\title{
Steadiness of a "water bell" surface to a destruction at a flow around of the thin rods assembly
}

\author{
Ekaterina Slesareva $^{1 \mathrm{a}}$, Ruslan Dekhtyar ${ }^{1}$, and Valeriy Ovchinnikov ${ }^{1}$ \\ ${ }^{1}$ SB RAS, Kutateladze Institute of Thermophysics, 630090 Novosibirsk, Russia
}

\begin{abstract}
The experimental research of hydrodynamic stability of a dome-shaped film liquid at a flow around a thin plate has been carried out. Experiments were carry out with a film in shape $\mathrm{a}$ «water bell». The film was formed by a leak-in jet of water width $10 \mathrm{~mm}$ on a hard disk with diameter $14.5 \mathrm{~mm}$. The width of a plate $\zeta$ changed from 0.05 to $3.5 \mathrm{~mm}$. The plate placed along or across relative to the vector of velocity of a liquid in a film. Experiments have shown, that stability of a film of liquid at a flow around the plate is defined by velocity of water and a thickness of a film $\delta$ in front of the rod. It is shown, that for the appointed value of Reynolds number $\mathrm{Re}_{\delta}$ probably continuous flow at a flow around the plate, if Weber number $\mathrm{We}_{\zeta}$ less than threshold value. The criterion of steadiness a film of the «water bell» by a surface destruction at a flow around the rod is determined on the transverse size of the rod relative to the vector of velocity of a liquid.
\end{abstract}

\section{Introduction}

Use a film of liquid in heat exchangers (condensers, absorbers, distillation columns, etc.), does of great importance study of such flow. The structure of the thin liquid film flow influences significantly the local and average characteristics of heat and mass transfer. It is known, that the intensification of heat and mass transfer in case of the developed wave surface occurs, both because of increase in the surface of heat and mass transfer, and owing to change of a velocity profile in a film $[1,2]$. One of forms a liquid film is dome-shaped films. Such films are formed at a leak-in a jet of liquid on a hard disk. The first works in which dome-shaped films of a liquid are study, it a «water bell», have been published in 1833 Felix Savart [3]. In works [4, 5] models of a «water bell» are submitted. Velocity of a liquid in film $U$ depending on distance $Y$ from a disk surface is presented by dependence

$$
U=\sqrt[2]{U_{0}^{2}+g \cdot Y},
$$

where $\mathrm{g}$ - gravity acceleration, $U_{0}$ - velocity on the brink of a disk. The thickness of a film $\delta$ at section $Y$ calculate in following form

$$
\delta=\frac{Q}{2 \pi \cdot R \cdot U}
$$

\footnotetext{
a Corresponding author: styuardessa@yandex.ru
} 
here $Q$ - the volumetric rate of flow of a liquid, $R$ - radius of a «water bell» profile in cross-section at altitude $Y$.

For creation of the developed wave surface the aggregated of thin rods used. At interacting a dome-shaped film flow with rod can be a surface destruction of liquid film. On fig. 1 demonstrate change of a regime flow when shift up on $20 \mathrm{~mm}$ assemblage of rods as for constant water flow rate. Diameter of the rod and a water flow rate is insufficient for definition of a flow regime. It is required to define the additional criteria, allowing to predict a flow regime considering a position of rod relative to a surface of a «water bell». The problem can be simplified if to observe conditions of stability for a thin plate in width $\zeta$. The work purpose is reception and the analysis of data on hydrodynamic stability of a dome-shaped film liquid at a flow around a thin plate.
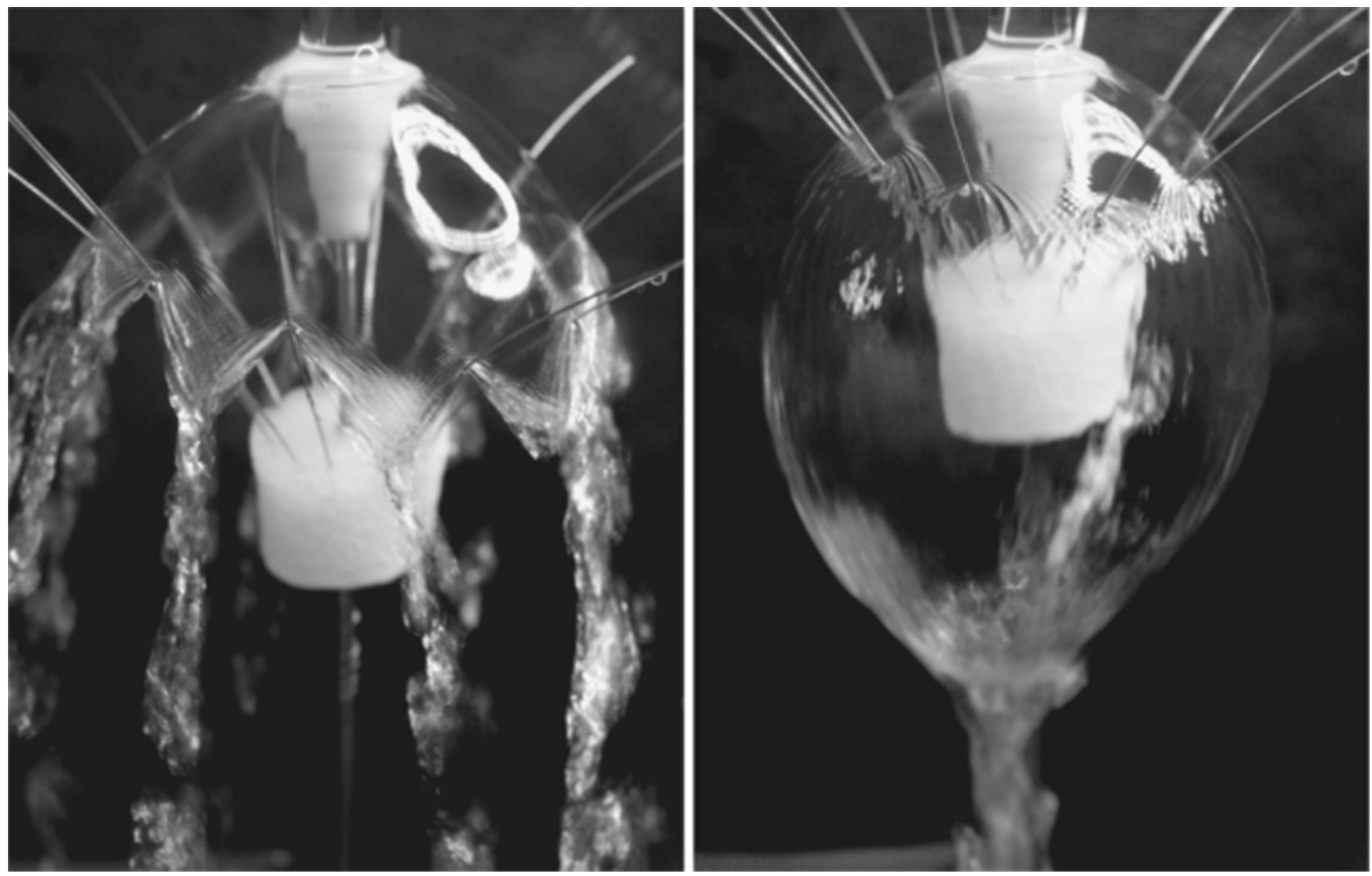

Figure 1. Influence of position of the aggregated 12 of thin rods relative to a surface of a «water bell» on a flow regime. Diameter rods $0.35 \mathrm{~mm}$, flow rate $Q=55 \mathrm{ml} / \mathrm{s}, U_{0}=0.74 \mathrm{~m} / \mathrm{s}$.

\section{A measurement procedure}

Experiments carry out with a «water bell», formed at a leak-in of a jet of water on a disk in diameter of $14.5 \mathrm{~mm}$. The jet was formed on an exit of a brass tube by a inside diameter of $10 \mathrm{~mm}$ on distances from 5 to $60 \mathrm{~mm}$ between a edge of a tube and a disk surface. The flow rate was measured on the calibrated glass tube in length of $400 \mathrm{~mm}$ and diameter of $9.5 \mathrm{~mm}$ by pressure drop. Calibrating the flow rate it was carried out by measurement of velocity of change of weight of the filled volume. Calibrating accuracy is $0.3 \mathrm{ml} / \mathrm{s}$. By of digital video cameras the photo-video shooting of pictures of a current with a speed to $300 \mathrm{fps}$ shoot a film. For research of agency of a plate width on a flow of a film of a liquid the wedge-shaped plate from stainless steel of $0.1 \mathrm{~mm}$ was used by thickness, width of a plate changed from 0.05 to $3.5 \mathrm{~mm}$ at length of $34.5 \mathrm{~mm}$. The size was defined in the area of intersection of a plate surface with a liquid film. In experiments the plate placed along or across relative to the vector of velocity of a liquid in a film. By means of the microscrew the plate moved on a normal to a film surface that has allowed defining precisely a size at the moment of destructing or rebuilding surface of a surface film liquid of a «water bell». 


\section{Discussion of results}

The experimental research has shown that on stability of a film liquid at interacting with a plate estimating a value render velocity $U$ and a thickness of a film $\delta$ in an intersection point. Values $U$ and $\delta$ are computed on dependences (1) and (2), accordingly. Processing of frames of a video filming of a «water bell» has shown, that dependence of radius profile $R$ from location $Y$ can be presented smooth functions in the following form:

$$
\delta=A \cdot Y^{a} \cdot \exp \left[\left(\frac{Y-C}{B}\right)^{b}\right]
$$

On fig. 2 are presented results comparisons of form a «water bell» with calculation on dependence (3) for various flow rate $Q$. For sizes in mm parameters $A, a, B, b$ in dependence (3) is: a) $A=5,9$; $a=0,5 ; B=164 ; b=8 ; C=-34 ; \mathbf{b}) A=3,9 ; a=0,5 ; B=132 ; b=12 ; C=-50$.
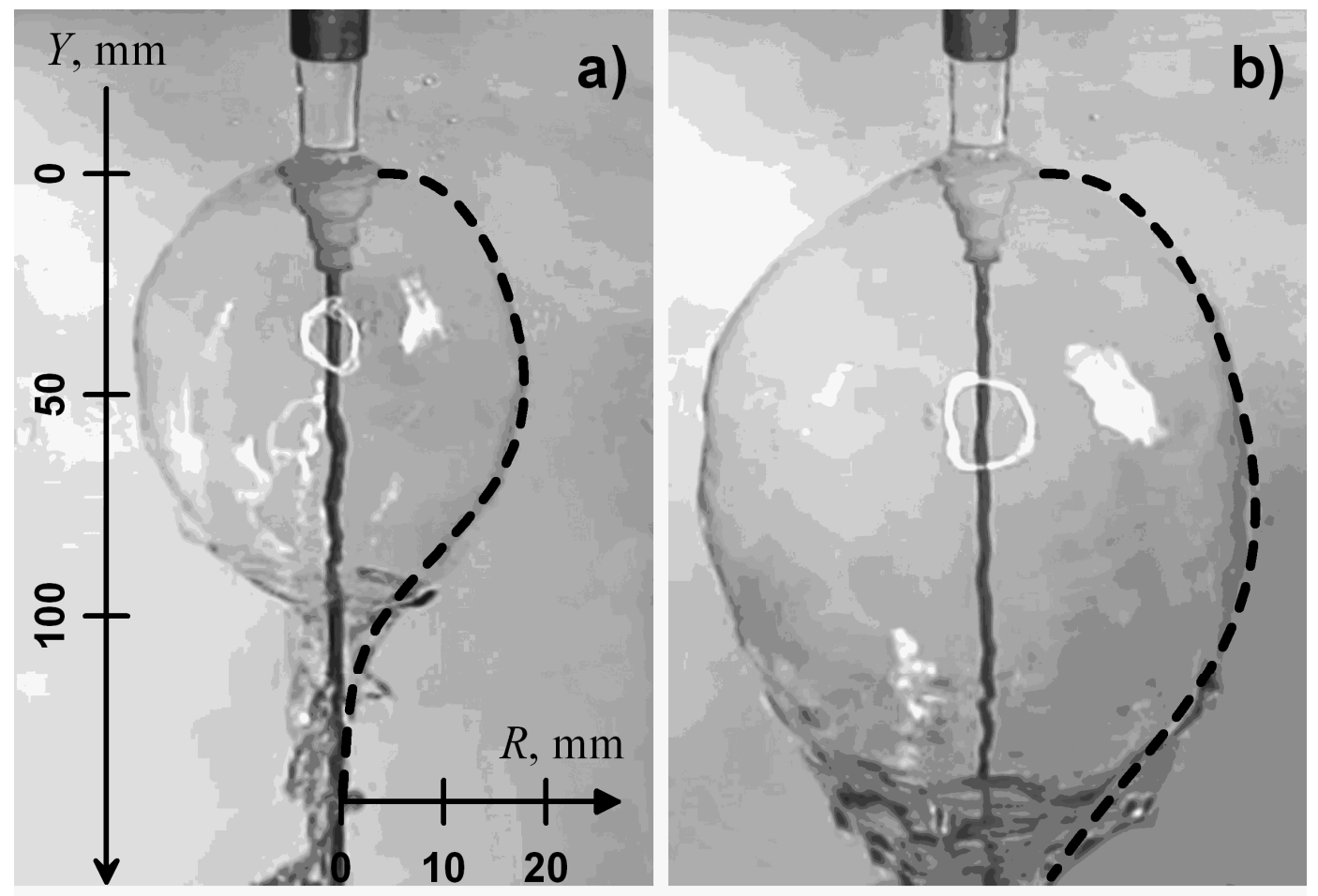

Figure 2. Frames of a video flow of dome-shaped film. Dashed line calculation $R(Y)$ on dependence (3); a) flow rate $Q=52 \mathrm{ml} / \mathrm{s}$; b) flow rate $Q=73 \mathrm{ml} / \mathrm{s}$.

Dependence (3) well presents a profile of a bell without waves on surface a film liquid. Using the obtained data on a rate of velocity $U$, a thickness of a film $\delta$, width of a plate $\zeta$ have been counted dimensionless criteria at the moment of destructing or rebuilding surface of a film liquid of a «water bell». It is Reynolds number $\operatorname{Re}_{\delta}=\frac{U \cdot \delta}{v}$ and Weber number $W e_{\xi}=\frac{\rho \cdot U^{2} \cdot \zeta}{\sigma}$, where $v$ - kinematics viscosity of the fluid, $\rho$ - density of the liquid, $\sigma$ - surface tension. The charts of the flow conditions of stability of a film in coordinates Reynolds number $\mathrm{Re}_{\delta}$ and Weber number $\mathrm{We}_{\zeta}$ are presented in fig. 3. At arrangement of a plate across in the direction of flow destructing surface film occurs at much smaller values of width of a plate $\zeta$, than at arrangement of a plate along, rebuilding surface film 
occurs at sizes close to a thickness of a film (a line 5). For arrangement of a plate along in the direction of flow rebuilding surface film occurs at values of width rather less, than at destructing.

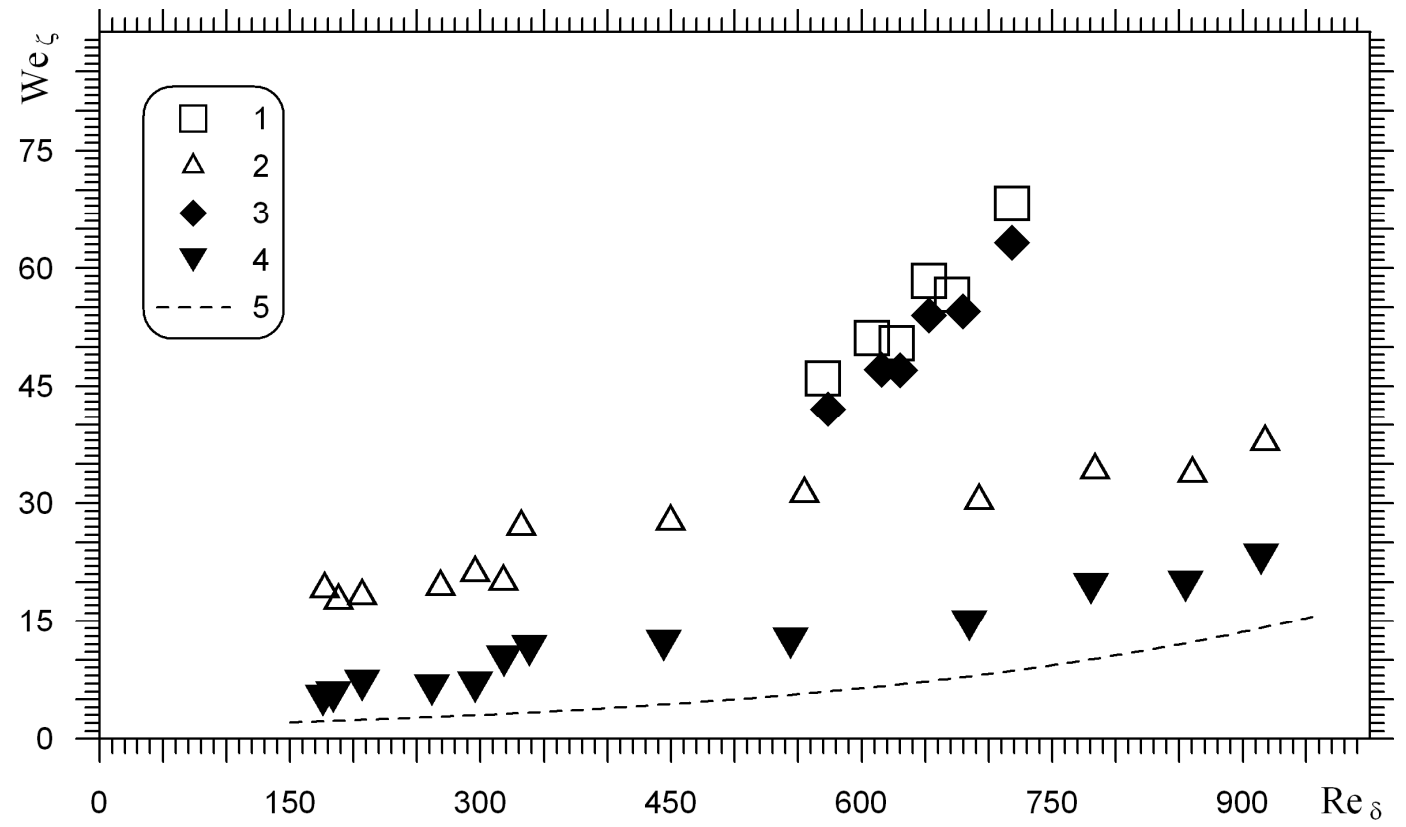

Figure 3. A charts of the flow conditions as function $\mathrm{We}_{\zeta}$ from $\mathrm{Re}_{\delta} .1$ - surface a film destruction (plate along in the direction of flow); 2 - surface destruction (plate across in the direction of flow); 3 - surface a film rebuilding (plate along in the direction of flow); 4 - surface a film rebuilding (plate across in the direction of flow); 5 Weber number $\mathrm{We}_{\zeta}$ at $\zeta=\delta$.

It is shown, that for the appointed value of Reynolds number $\mathrm{Re}_{\delta}$ probably continuous flow at a flow around the plate, if Weber number $\mathrm{We}_{\zeta}$ less than threshold value. The criterion of steadiness a film of the «water bell» by a surface destruction at a flow around the rod is determined on the transverse size of the rod relative to the vector of velocity of a liquid.

Acknowledgements. This work was done under a grant from the Russian Science Foundation N 14-19-00352.

\section{References}

1. S.V. Alekseenko, V.E. Nakoryakov, B.G. Pocusaev Wave Flow of Liquid Films (Begell house, New York, 1994)

2. V.E. Nakoryakov, S.Y. Misyura, S.L. Elistratov, J. Engin. Therm., 22(1), 1, (2013)

3. F. Savart, Ann. De Chim.,. 54, 56, (1833)

4. G. Taylor, Proc. R. Soc. London A, 253(13), 289, (1959)

5. J.Y. Parlange, J. Fluid Mech., 29(2), 361, (1967) 